\title{
MRI Characteristics of Primary Tumors and Metastatic Lesions in Molecular Subgroups of Pediatric Medulloblastoma: A Single-Center Study
}

\author{
(DD. Mata-Mbemba, DM. Zapotocky, DS. Laughlin, (D) M.D. Taylor, DV. Ramaswamy, and (D). Raybaud
}

\begin{abstract}
BACKGROUND AND PURPOSE: Molecular grouping of medulloblastoma correlates with prognosis and supports the therapeutic strategy. We provide our experience with the imaging features of primary and metastatic disease in relation to the molecular groups.

MATERIALS AND METHODS: One hundred nineteen consecutive patients (mean age, $7.3 \pm 3.8$ years at diagnosis; male, 79 [66.4\%]) with a confirmed diagnosis of medulloblastoma and interpretable pretreatment MRIs were retrieved from our data base from January 2000 to December 2016. Each patient was assigned to wingless, sonic hedgehog, group 3, or group 4 molecular groups. Then, we determined the imaging features of both primary and metastatic/recurrent disease predictive of molecular groups.

RESULTS: In addition to recently reported predictors based on primary tumor, including cerebellar peripheral location for sonic hedgehog (adjusted odds ratio $=9, P<.0001$ ), minimal enhancement of primary group 4 tumor (adjusted odds ratio $=5.2, P<.0001$ ), and cerebellopontine angle location for wingless (adjusted odds ratio $=1.4, P=.03$ ), ependymal metastasis with diffusion restriction and minimal postcontrast enhancement ("mismatching pattern") (adjusted odds ratio $=2.8, P=.001$ ) for group 4 and spinal metastasis for group 3 (adjusted odds ratio $=1.9, P=.01$ ) also emerged as independent predictors of medulloblastoma molecular groups. Specifically, the presence of a metastasis in the third ventricular infundibular recess showing a mismatching pattern was significantly associated with group $4(P=.02)$.
\end{abstract}

CONCLUSIONS: In addition to imaging features of primary tumors, some imaging patterns of metastatic dissemination in medulloblastoma seem characteristic, perhaps even specific to certain groups. This finding could further help in differentiating molecular groups, specifically groups 3 and 4 , when the characteristics of the primary tumor overlap.

ABBREVIATIONS: $\mathrm{aOR}=$ adjusted odds ratio; $\mathrm{CPA}=$ cerebellopontine angle; $S H H=$ sonic hedgehog; $W N T=$ wingless

M edulloblastoma is a highly malignant tumor (World Health Organization grade IV) and is also the most common malignant posterior fossa tumor in children. It has a high propensity for leptomeningeal spread. Approximately 20\%-

Received November 1, 2017; accepted January 2, 2018.

From the Department of Diagnostic Imaging (D.M.-M., S.L., C.R.), Division of Neurooncology (M.Z., V.R.), and Department of Neurosurgery (M.D.T.), Hospital for Sick Children, University of Toronto, Toronto, Ontario, Canada.

Daddy Mata-Mbemba and Michal Zapotocky are co-first authors and contributed equally to this work.

Vijay Ramaswamy and Charles Raybaud are co-senior authors and contributed equally to this work.

Previously presented, in part, orally at: Annual Meeting of the American Society of Neuroradiology and the Foundation of the ASNR Symposium, April 33-27, 2017; Long Beach, California.

Please address correspondence to Charles Raybaud, MD, Department of Radiology, Hospital for Sick Children, 555 University Ave, Toronto, ON, M5G 1X8, Canada; e-mail: charles.raybaud@sickkids.ca

三 Indicates article with supplemental on-line tables.

http://dx.doi.org/10.3174/ajnr.A5578
$35 \%$ of patients present with metastatic disease at diagnosis ${ }^{1-3}$; and $30 \%-50 \%$, late during the treatment/surveillance period. ${ }^{4}$ In 1 series of 86 patients with medulloblastoma, 19 (22.1\%) patients had metastases at diagnosis and $22(25.6 \%)$ patients had metastases late during the surveillance period. ${ }^{5}$

Recent molecular studies have shown that medulloblastoma is not a single entity but rather a constellation of groups, each with a distinct developmental origin, meaning that each may therefore benefit from subgroup-specific treatments. ${ }^{6-8}$ These groups are wingless (WNT), sonic hedgehog $(\mathrm{SHH})$, group 3, and group 4. ${ }^{3,7,8}$ However, high cost and lack of access to this molecular grouping in most centers in the world constitute a main limitation in its routine clinical use to guide treatment. MR imaging of the brain and spine is however routinely performed in all patients with medulloblastoma before treatment to characterize the primary tumor, to aid with surgical neuronavigation, and to assess intracranial and spinal metastases. The presence of the latter worsens the prognosis 
and would upstage therapeutic strategies from average risk to high risk. ${ }^{9}$

A handful of recent reports based on a smaller number of patients investigated the possibility of using imaging features of the primary tumor on MR imaging as surrogate markers for molecular groups. ${ }^{10,11}$ None of them have evaluated the relationship between imaging features of metastatic disease and molecular grouping.

The aim of this single-institution study was 2 -fold: 1 ) to validate the predictive value of imaging features of primary tumor with respect to their molecular group assignment, and 2) to test the hypothesis that imaging patterns of metastatic disease spread also correlate with the molecular groups.

\section{MATERIALS AND METHODS}

\section{Patients}

After institutional review board approval and a waiver of consent, 119 consecutive patients (mean age at diagnosis, $7.3 \pm 3.8$ years; male, 79 [66.4\%]) with a confirmed diagnosis of medulloblastoma and interpretable pretreatment MRIs were retrieved from the data base at the Hospital for Sick Children, Toronto, Canada, during a 17-year period (from January 2000 to December 2016). The pathologic diagnosis and the subtypes (large-cell anaplastic, classic, or desmoplastic) of the primary tumor based on hematoxylineosin-stained slides of formalin-fixed paraffin-embedded material were also available in our data base for all 119 included patients. Patients referred from outside hospitals without an initial MR imaging study available were excluded.

\section{Molecular Analysis}

The 4 molecular groups (WNT, SHH, group 3, and group 4) of medulloblastoma were determined using NanoString nCounter system (nanoString Technologies, Seattle, Washington), as previously described from both formalin-fixed paraffin-embedded tissue and frozen tissue. ${ }^{7,12,13}$ Molecular grouping data were available for all 119 patients.

\section{MR Imaging}

All patients underwent brain and spine MR imaging at either 1.5 or $3 \mathrm{~T}$ on different magnets and using different protocols during the 17-year period. The MR imaging protocol used presently for brain tumor in our institution includes at least the sequences shown in On-line Tables 1 and 2.

Most patients included in this study underwent DWI, except for $24(20.2 \%)$ patients who were examined before the implementation of DWI in our routine MR imaging protocol.

\section{Imaging Analysis of the Primary Tumor}

A neuroradiology fellow and 2 attending neuroradiologists reviewed the MR images. Before starting the review, the 3 readers defined the imaging features to be assessed, including the location and the degree of enhancement of the primary tumor. Ten randomly selected cases were assessed by each reviewer to refine and standardize the definition of these parameters. Afterward, 2 of these readers independently reviewed the MR images blinded to clinical, pathologic, and molecular data. For discordant readings between the 2 first readers, the third reader reviewed the cases and a consensus was reached among the 3 readers at the end. The initial results of the 2 first readers were used to generate the interreader agreement.

The readers evaluated the following MR imaging features: tumor location in relation to the fourth ventricle, tumor size, tumor margin, diffusion restriction, enhancement pattern, cyst/cavitation change, hemorrhage or calcification, the presence of peritumoral vasogenic edema, and the presence of supratentorial hydrocephalus.

The tumor location was categorized as follows: 1) fourth ventricle, which was further divided in 3 groups: fourth ventriclemidline, fourth ventricle with unilateral extension along the lateral recess, and fourth ventricle with bilateral extension along the lateral recesses; 2) cerebellopontine angle (CPA); 3) cerebellar periphery, which was defined as tumor involving the cerebellar cortex; and 4) paraventricular region, which was defined as tumor located close to the fourth ventricle in the deep cerebellar white matter without extending into it. The tumor size was a mean of the 3 orthogonal diameters of the primary tumor. The tumor margin was characterized as ill-defined if $>50 \%$ of the margin could not be distinguished from the surrounding cerebellar parenchyma on the basis of all imaging sequences. ${ }^{11}$ The diffusion restriction was characterized by the presence of any low signal area within the tumor on ADC maps, which was determined visually. The degree of diffusion restriction was quantified by ROI-based ADC analysis following the approach of Yeom et al. ${ }^{14}$ The degree of tumor enhancement was defined as one of the following: 1) none or minimal (if less than one-third of the mass was enhanced), 2) incomplete (between one-third and two-thirds of the mass was enhanced), and 3) diffuse (if more than two-thirds of the mass was enhanced). Hemorrhage or mineralization was defined as foci of blooming on $2 \mathrm{D}$ gradient recalled-echo sequence.

\section{Imaging Analysis of Metastatic Disease}

Blinded to molecular and pathologic data, a neuroradiology fellow and another attending neuroradiologist reviewed the imaging features of metastatic disease, which were defined as early (at diagnosis, ie, before the treatment including chemotherapy, radiation, and/or an operation) or late/recurrent (after treatment initiation including chemotherapy, radiation, and/or an operation) metastases, which were further characterized as ependymal or leptomeningeal. Ependymal metastases were searched specifically along the ependyma of the lateral and third ventricles, especially in the third ventricular infundibular recess. The recurrent tumor was searched at the site of primary tumor, including within the fourth ventricle. Leptomeningeal metastases were divided into supratentorial, infratentorial, and spinal. Furthermore, signal patterns of all intracranial metastatic disease were evaluated and characterized as diffusion restricting or not and as postcontrast enhancing or not. Imaging characteristics of metastases were called "mismatching pattern" when the metastatic lesion showed diffusion restriction but no/minimal postcontrast enhancement and "matching pattern" when the lesion showed both diffusion restriction and postcontrast enhancement. Consensus was used to resolve disagreement among the readers. 
Table 1: Patient demographics with respect to molecular groups

\begin{tabular}{|c|c|c|c|c|c|c|}
\hline Parameters & WNT $(n=15)(\%)$ & $S H H(n=26)(\%)$ & Group $3(n=29)(\%)$ & Group $4(n=49)(\%)$ & Total $(n=119)(\%)$ & $P$ \\
\hline Sex & & & & & & $.02^{\mathrm{a}}$ \\
\hline Male & $7(46.7)$ & $13(50)$ & $20(69)$ & $39(80)$ & 79 (66.4) & \\
\hline Female & $8(53.3)$ & $13(50)$ & $9(31)$ & $10(20)$ & $40(33.6)$ & \\
\hline Age at diagnosis $(y r)^{b}$ & $8.7(3.1)$ & $6.2(4.6)$ & $6.1(3.8)$ & $8.1(3.2)$ & $7.3(3.8)$ & $<.0001^{\mathrm{a}}$ \\
\hline Histology & & & & & & $.02^{\mathrm{a}}$ \\
\hline LCA & $1(6.7)$ & $9(34.6)$ & $13(44.8)$ & 7 (14.3) & $30(25.2)$ & \\
\hline Classic & $14(93.3)$ & $8(30.8)$ & $15(51.7)$ & $39(79.6)$ & $76(63.9)$ & \\
\hline Desmoplastic & $0(0)$ & $9(34.6)$ & $1(3.5)$ & $3(6.1)$ & 13 (10.9) & \\
\hline
\end{tabular}

Note:-LCA indicates large-cell anaplastic.

a Statistically significant.

${ }^{b}$ Mean with SDs.

Table 2: Locations of primary tumors with respect to molecular groups

\begin{tabular}{lcccccccc}
\hline & \multicolumn{9}{c}{ Fourth Ventricle } & & \\
\cline { 2 - 6 } Subgroup & $\begin{array}{c}\text { No. } \\
(\boldsymbol{n}=119)(\%)\end{array}$ & $\begin{array}{c}\text { Midline-Vermis } \\
(\boldsymbol{n}=\mathbf{4 0})(\%)\end{array}$ & $\begin{array}{c}\text { 4th-uniCPA } \\
(\boldsymbol{n}=\mathbf{2 5})(\%)\end{array}$ & $\begin{array}{c}\text { 4th-biCPA } \\
(\boldsymbol{n}=17)(\%)\end{array}$ & $\begin{array}{c}\text { Subtotal } \\
(\boldsymbol{n}=\mathbf{8 1})(\%)\end{array}$ & $\begin{array}{c}\text { CPA } \\
(\boldsymbol{n}=\mathbf{6})(\%)\end{array}$ & $\begin{array}{c}\text { Paraventricular } \\
(\boldsymbol{n}=\mathbf{1 2})(\%)\end{array}$ & $\begin{array}{c}\text { Cerebellar Periphery } \\
(\boldsymbol{n}=19)(\%)\end{array}$ \\
\hline WNT & $15(12.6)$ & $5(12.5)$ & $3(12)$ & $1(5.9)$ & $8(9.9)$ & $3(50)$ & $3(25)$ & $0(0)$ \\
SHH & $26(21.8)$ & $3(7.5)$ & $0(0)$ & $0(0)$ & $3(3.7)$ & $3(50)$ & $2(16.7)$ & $18(94.7)$ \\
Group 3 & $29(24.4)$ & $11(27.5)$ & $8(32)$ & $6(35.3)$ & $25(30.9)$ & $0(0)$ & $4(33.3)$ & $0(0)$ \\
Group 4 & $49(41.2)$ & $21(52.5)$ & $14(56)$ & $10(58.8)$ & $45(55.5)$ & $0(0)$ & $3(25)$ & $1(5.3)$ \\
\hline
\end{tabular}

Note:-4th-uniCPA indicates fourth ventricle with unilateral extension along the lateral recess toward the CPA; 4th-biCPA, fourth ventricle with bilateral extension along bilateral recesses toward the CPAs.

\section{Statistics and Analysis}

First, we evaluated the relationship between the molecular groups and MR imaging features of primary or metastatic disease using the Fisher exact test. Afterward, to test the overall performance of MR imaging patterns of metastatic disease spread in predicting the molecular grouping, we conducted a stepwise multivariable multinomial logistic regression in which variables related to the patterns of metastatic disease spread were put in the model along with the previously reported predictors (ie, MR imaging features of primary tumors previously reported as independent predictors of molecular grouping, including CPA location for WNT, minimal enhancement of group 4 tumors, and cerebellar peripheral location for $\mathrm{SHH}$ ) to determine the overall independent predictors of molecular grouping. All statistical analyses were performed using JMP Pro version 13 software (SAS Institute, Cary, North Carolina), and $P$ values $<.05$ were considered statistically significant.

\section{RESULTS}

\section{Patients}

The results of our patients, including the distribution of their demographic data and pathologic subcategorization of the primary tumor, are shown in Table 1.

Of the 119 patients, NanoString assay revealed 15 WNT (12.6\%), $26 \mathrm{SHH}(21.8 \%), 29$ group 3 (24.4\%), and 49 group 4 (41.2\%) medulloblastoma molecular groups. $\mathrm{SHH}$ and group 3 patients were younger $(P=.02)$. Male patients were predominant $(P=.02)$.

\section{Primary Tumor}

There was an almost perfect (reference, 0.81-1.0) agreement among the readers in determining both the location $(\kappa=86 \%)$ and the degree of enhancement $(\kappa=82 \%)$ of the primary tumor. ${ }^{10}$

Depending on the group, tumor locations were highly associated with molecular groups. Their distribution is shown in

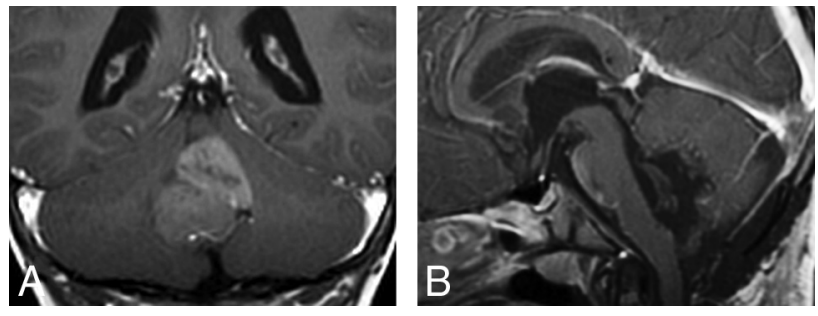

FIG 1. Coronal postcontrast TIWI (A) showing SHH tumor within the fourth ventricle. The postoperative sagittal postcontrast $T 7(B)$ confirmed that the tumor was arising from the flocculonodular lobe.

Table 2. In 20\% (3/15), WNT tumors were strictly limited to the CPA, but this was not exclusive to WNT because 3 (11.5\%) of 26 $\mathrm{SHH}$ tumors were also centered in the CPA. Four of 15 WNT tumors were centered in the fourth ventricle while extending laterally along 1 (in 3 cases) or both (in 1 case) lateral recesses. This location was also observed in groups 3 and 4 . The cerebellar periphery (cerebellar cortex) was the primary location of $69 \%$ of SHH tumors $(P<.0001)$; this corresponds to a positive predictive value of $94.7 \%$ (95\% CI, 84\%-100\%) for SHH. Three $S H H$ tumors were located in the fourth ventricular midline (Fig 1), but none them extended from the fourth ventricle into the CPA via the lateral recess $(P=.0002)$.

Groups 3 and 4 were primarily located in the fourth ventricle $(P<.0001)$ and remained within the midline of the fourth ventricle in $80 \%$ of cases $(P=.02)$. Of the 12 tumors noted in paraventricular locations, 4 (33.3\%) were group 3, but this association was not statistically significant $(P=.45)$.

There was a statistically significant difference in the degree of primary tumor enhancement among molecular groups $(P<.0001)$ that is, minimal enhancement was noted in $55.1 \%$ of group 4 patients $(P<.0001)$, showing a positive predictive value of $75 \%$ (95\% CI, $60 \%-89 \%)$. Only 4 (13.8\%) group 3 patients demonstrated minimal enhancement. On the other hand, $86.3 \%$ of $\mathrm{SHH}$ tumors demonstrated strong avid enhancement $(P<.0001)$.

AJNR Am J Neuroradiol 39:949-55 May 2018 www.ajnr.org 951 
No significant statistical difference was found among the molecular groups regarding the following tumor characteristics: tumor margin, ADC value, cystic change/necrosis, hemorrhage or calcification, the presence of a peritumoral vasogenic edema, and the presence of supratentorial hydrocephalus $(P>.05)$.

\section{Metastatic Disease}

Of the 119 patients, 34 patients $(28.6 \%)$ were diagnosed with metastatic disease, including 25/34 (73.5\%) initial and 9/34 (26.5\%) later metastases. These metastatic diseases were distributed as follows: 1 of 15 WNT (6.7\%) (none at diagnosis, 1 late); 2 of $26 \mathrm{SHH}$ (7.7\%) (2 late), 13 of 29 group 3 (44.8\%) (8 at diagnosis, 5 late); 15 of 49 group $4(30.6 \%)$ (14 at diagnosis, 1 late). In 3 cases, multi-

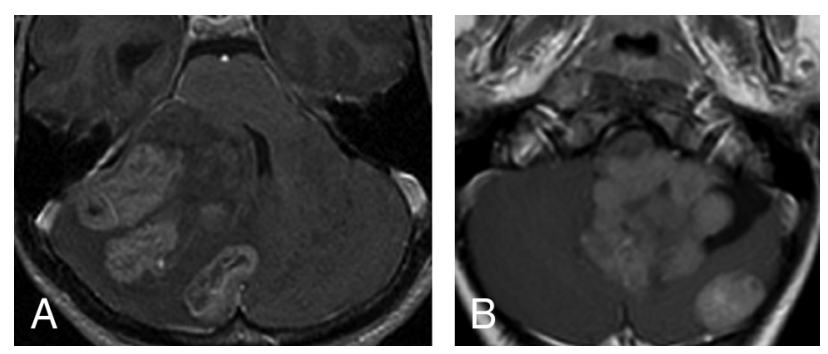

FIG 2. Multifocal (synchronous) SHH tumors at diagnosis. The multiple tumors on this axial postcontrast $\mathrm{Tl}$ are located in, and presumably originate within, the cerebellar cortex $(A)$. In the second patient $(B)$, there is 1 large fourth ventricular mass with an associated separate cortical mass posteriorly. In this case, the main tumor is lobulated and appears more like a conglomerate of multiple masses.

Table 3: Imaging features of initial metastases with respect to their molecular group assignment $^{\mathrm{a}}$

\begin{tabular}{lccccc}
\hline & \multicolumn{5}{c}{ Initial Metastasis $(\boldsymbol{n}=\mathbf{2 5})$} \\
\cline { 2 - 6 } \multicolumn{1}{c}{$\begin{array}{c}\text { WNT } \\
\text { MRI Signals }\end{array}$} & $\begin{array}{c}\text { SHH } \\
(\boldsymbol{n}=\mathbf{0})(\%)\end{array}$ & $\begin{array}{c}\text { Group 3 } \\
(\boldsymbol{n}=3)(\%)\end{array}$ & $\begin{array}{c}\text { Group 4 } \\
(\boldsymbol{n}=\mathbf{8})(\%)\end{array}$ & $(\boldsymbol{n}=14)(\%)$ & $\boldsymbol{P}$ Value \\
\hline Ependymal C-/D+ & $0(0)$ & $0(0)$ & $(0)$ & $6(42.8)$ & $.01^{\mathrm{b}}$ \\
3rd V.I.R, C-/D+d & $0(0)$ & $(0)$ & $(0)$ & $4(28.6)$ & $.02^{\mathrm{c}}$ \\
Ependymal C+/D+ & $0(0)$ & $0(0)$ & $3(37.5)$ & $0(0)$ & $.02^{\mathrm{c}}$ \\
Leptomeningeal C-/D+ & $0(0)$ & $0(0)$ & $0(0)$ & $3(21.4)$ & .05 \\
Leptomeningeal C+/D+ & $0(0)$ & $3(100)$ & $5(62.5)$ & $5(35.7)$ & .17 \\
Leptomeningeal C+/D- & $0(0)$ & $0(0)$ & $0(0)$ & $4(28.6)$ & $.02^{\mathrm{c}}$ \\
\hline
\end{tabular}

Note:- C - indicates no contrast enhancing; D+, diffusion-restricting; $D-$, no diffusion-restricting; $C+$, contrastenhancing; 3rd V.I.R, third ventricle infundibular recess.

a The sum of metastases in some locations is higher than the total number of patients in the group because each case could have multiple metastases in $>1$ compartment.

${ }^{b} P$ value determined using the Fisher exact test.

c Statistically significant.

${ }^{\mathrm{d}}$ Subgroup of patients with mismatching ependymal metastasis in the third ventricle infundibular recess.
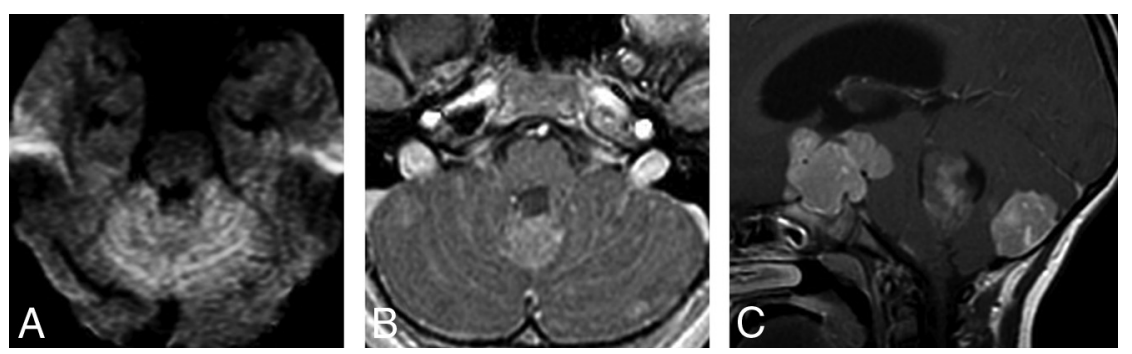

FIG 3. Metastatic group 3 tumor. In the first patient ( $A$ and $B$ ), the leptomeningeal "sugar coating" metastases demonstrate both diffusion restriction $(A)$ and leptomeningeal enhancement on an axial postcontrast $T 1$ image $(B)$. In the second patient, the primary tumor appears smaller than its leptomeningeal suprasellar, avidly enhancing metastasis (C). An avidly enhancing nodular metastasis involving the cortex in the cerebellum posteriorly is also noted. nodular SHH tumors were initially diagnosed at presentation as theses; it is now thought that this pattern may rather reprelar cortex (Fig 2). This feature distinguished $\mathrm{SHH}$ from

\section{Initial Metastasis} Table 3.

In group 3, metastases demonstrated a matching pattern regardless of their ependymal and leptomeningeal locations and the primary tumor was smaller than the largest metastatic tumor $(P=.01)$ (Fig $3 C)$. Seven $(87.5 \%)$ of the 8 group 3 patients showed initial spinal metastases $(P=.02)$.

In group 4, of the 14 patients with initial metastasis, 6 (42.9\%) showed ependymal metastases with a mismatching pattern (Table 3 and Fig $4 A,-B$ ). Four of these 6 patients showed a mismatching metastatic lesion in the ependyma of the third ventricular infundibular recess, a finding that was specific for group $4(P=.02)$ (Fig $4 C$ ), corresponding to a positive predictive value of $100 \%$ for group 4 . One group 4 patient (7.1\%) showed an ependymal metastasis, which did not restrict or enhance.

On the other hand, leptomeningeal metastases were seen in 12 $(85.7 \%)$ of 14 group 4 patients and demonstrated 3 different imaging patterns: metastases showing a matching pattern in 5 patients, a mismatching pattern in 3 patients, and postcontrast enhancement and no diffusion restriction in $4 \mathrm{pa}$ tients. This mixture of patterns of leptomeningeal metastases was significantly associated with group $4(P=.0002)$ only.

\section{Late Metastases/Recurrence}

Of the 9 patients with late metastasis, local tumor recurrence was seen in only 1 group 4 patient, who showed a mass lesion within the surgical bed (Fig $5 A,-B$ ). In the 8 remaining patients, recurrent metastatic disease in the posterior fossa presented as multiple scattered lesions.

One patient in the WNT group had ependymal metastases that demonstrated diffusion restriction and postcontrast enhancement, with an enhancing leptomeningeal metastasis in the anterior interhemispheric fissure. Of the 2 patients in the SHH group, one showed only 1 spinal metastasis, while the other showed multiple supra- and infratentorial leptomeningeal metastases.

Five of the 9 late metastases were from group $3(P=.02)$. Four $(80 \%)$ of these 5 

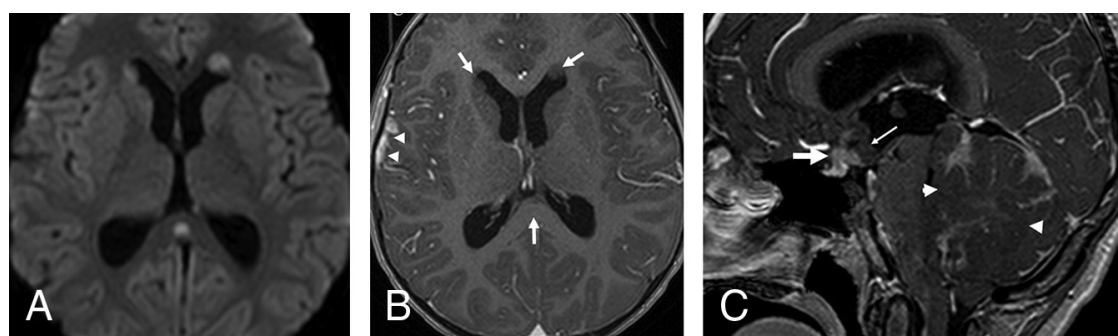

FIG 4. Metastatic group 4 tumors. In the first patient ( $A$ and $B$ ), the ependymal metastases seen in the anterior horns of the lateral ventricles and in the splenium of corpus callosum demonstrate diffusion restriction $(B)$ without enhancement on contrast-enhanced $\mathrm{Tl}$ (arrows, B), while the leptomeningeal metastasis seen along the right temporal lobe demonstrates enhancement without appreciable diffusion restriction (arrowheads, $B$ ). In the second patient (C), the primary tumor demonstrates minimal enhancement on the contrast-enhanced $\mathrm{Tl}$ (arrowheads). The patient has a metastatic tumor in the suprasellar region with 2 components: One is ependymal in the infundibular recess and shows no enhancement (thin arrow), while the second is leptomeningeal and enhances strongly (thick arrow) like other metastasis in the posterior fossa, or supratentorially.
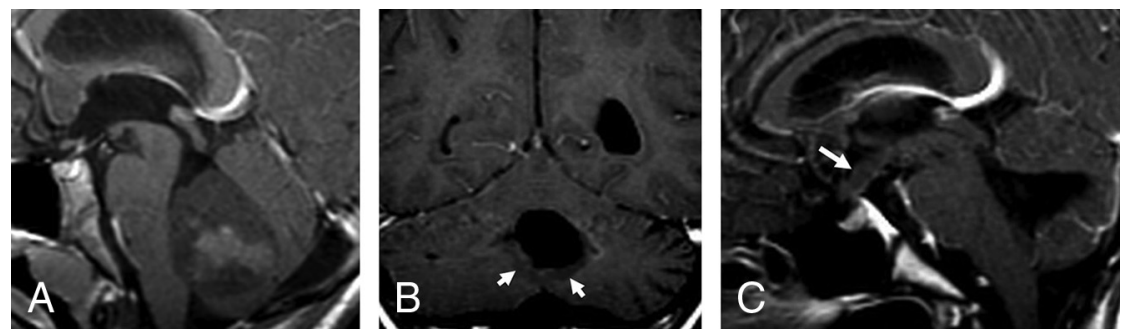

FIG 5. A recurrent group 4 tumor. $A$, The primary tumor demonstrates minimal postcontrast enhancement. Four years later, a recurrent tumor appeared along the lower margin of the postoperative cavity (arrows, $B$ ) as well as a metastasis in the infundibular recess and along the tuber cinereum (arrow, $C$ ), both of which are nonenhancing.

patients had spinal metastases $(P=.003)$. Of the 5 group 3 patients with late metastases, $4(80 \%)$ showed leptomeningeal and ependymal metastases demonstrating a matching pattern $(P=.003)$.

One group 4 patient (the same patient described above who had a local recurrence within the fourth ventricle) showed a late single metastasis in the third ventricular infundibular recess, demonstrating a mismatching pattern (Fig 5C), as well as diffuse spinal intradural extramedullary and intramedullary metastases.

\section{Overall Predictors of Molecular Groups}

The stepwise multivariable multinomial logistic regression model revealed that in addition to recently reported predictors based on primary tumor, including cerebellar peripheral location for $\mathrm{SHH}$ (adjusted odds ratio $[\mathrm{aOR}]=9, P<.0001$ ), minimal enhancement of primary group 4 tumor $(\mathrm{aOR}=5.2, P<.0001)$, and $\mathrm{CPA}$ location for WNT ( $\mathrm{aOR}=1.4, P=.03$ ), ependymal metastasis with diffusion restriction and minimal postcontrast enhancement (mismatching pattern) $(\mathrm{aOR}=2.8, P=.001)$ for group 4 and spinal metastasis for group $3(\mathrm{aOR}=1.9, P=.01)$ also emerged as independent predictors of medulloblastoma molecular groups. Specifically, the presence of a metastasis in the third ventricular infundibular recess showing mismatching pattern was significantly associated with group $4(P=.02)$.

\section{DISCUSSION}

In this article, we evaluated the relationship between the molecular groups and the imaging features of both primary and metastatic pediatric medulloblastoma tumors and found that some imaging features of these tumors are reliable in predicting the molecular groups. For the primary tumors, for instance, we validated the previous reports $^{11}$-that is, $\mathrm{SHH}$ tumors were mainly located in the cerebellar periphery with strong postcontrast enhancement, and group 4 tumors were located mainly in the fourth ventricles with usually minimal enhancement. However, in our series, only a small proportion (3/ $15,20 \%)$ of WNT tumors were truly located in the CPA; the CPA site was not specific for WNT tumors because $\mathrm{SHH}$ tumors may develop in the same location when they originate from the flocculus (2/26) (Fig 1).

Our findings are concordant with the report by Patay et al, ${ }^{10}$ who found that most WNT tumors were close to the midline but might lateralize toward the CPA. Gibson et $\mathrm{al}^{15}$ stated that WNT tumors develop in the midline within the fourth ventricle, while on the contrary, Perreault et $\mathrm{al}^{11}$ reported a positive predictive value of $100 \%$ for CPA tumors being WNT. Indeed, the lateral recess contains the portion of the lower rhombic lip, which has a strong WNT expression. ${ }^{16}$ The tumor might originate from there and extend either laterally to the CPA or, more often, develop medially to finally appear centered within the fourth ventricle, while other tumors centered in the fourth ventricle may extend laterally into 1 lateral recess or both.

Most (69.2\%) SHH tumors were located at the periphery of the cerebellar hemisphere. This result matches the existing literature that states that $\mathrm{SHH}$ tumors originate from the glutamatergic neuron precursor granule cells of the upper rhombic lip and form the external granular layer, a secondary proliferative zone that persists on the surface of the cerebellum until the second year. ${ }^{17}$ SHH tumors therefore develop from the cerebellar cortex and are commonly associated with a leptomeningeal desmoplastic reaction. Most develop in the cerebellar convexity of the hemispheres and vermis, but they may also develop from the flocculus, which is located within the CPA, or from the nodulus, which is located within the fourth ventricle. ${ }^{16}$ In our patients, $30.8 \%$ of $\mathrm{SHH}$ tumors were located either in the CPA or the lumen of the fourth ventricle. Even in atypical locations, $\mathrm{SHH}$ tumors may still be distinguished from the other groups because they commonly present with a very strong postcontrast enhancement (seen in $86.3 \%$ of $S H H$ tumors), which is thought to be due to leptomeningeal desmoplasia, which is associated with this cortical location. Another distinguishing feature of the $\mathrm{SHH}$ group tumor is the multinodularity and/or synchronous multifocal tumors at presentation. In 3 cases, patients presented with cortical cerebellar masses that were associated with several large cortical nodules scattered in other parts of the cerebellum, initially considered as metastases present at diagnosis. However, these may, in fact, rep- 
resent metachronous primary tumors rather than metastatic dissemination. In our data, this feature appears to be specific for the $\mathrm{SHH}$ group because nothing comparable was observed in the other groups.

In our series, most of the group 3 and 4 medulloblastomas were located in the fourth ventricular midline $(89.7 \%)$ or, uncommonly, in a paraventricular location (9\%). In $25 \%$ of the cases, the primary tumor in group 3 was small, possibly smaller than their largest metastasis, and then was unassociated with hydrocephalus. This finding suggests an aggressive malignancy with early metastases in a "young" tumor that did not give the ventricles time to expand. One may speculate that metastatic dissemination in group 3 could be an early clonal event, meaning that the tumor metastasizes earlier during its growth, which is consistent with the very poor prognosis of group 3 patients compared with other groups. ${ }^{18,19}$ Regarding tumors of group 4, they typically show no or only minimal enhancement, as was reported by Perreault et al. ${ }^{11}$ This contrasts with the usual demonstration in these tumors of prominent intratumoral vessels, consistent with high perfusion; it reflects a preserved blood-tumor barrier, an unexpected finding for a highly malignant embryonal tumor. This minimal or lack of enhancement, though not absolutely specific (found in $18.8 \%$ of group 3 tumors as well), is still a good identification mark of group 4 as opposed to other groups.

Regarding metastases, group 3 metastases showed a matching pattern (restricting and enhancing) regardless of their ependymal or leptomeningeal location. This contrasted with most (but not all) ependymal metastases of group 4 tumors, which showed a mismatching pattern (restricting but not enhancing), while most of their leptomeningeal metastases enhanced. The biologic meaning of this finding is not known.

Evaluation of late metastases also demonstrated different features for different molecular groups. Most the late metastatic disease belonged to group 3 and spread away from the primary site into the spinal theca. On the other hand, only 1 of the group 4 patients showed late metastases, including a local recurrence within the surgical site and metastasis in the third ventricular recess. Also, only 1 patient with a WNT tumor and 2 with $S H H$ tumors showed late metastases; this finding is consistent with the less malignant character of the tumors of these groups compared with group $3 .^{18,19}$

Third ventricular metastases have been known for a long time to occur in medulloblastoma. ${ }^{20}$ Most reports are case reports published before the era of molecular grouping, some of which were based on CT images, making it difficult to differentiate leptomeningeal from intraventricular masses. ${ }^{21-25}$ The description of Shelton et $\mathrm{al}^{23}$ resembles what we noted in at least 1 of our patients (Fig 4C): a suprasellar metastasis with an enhancing leptomeningeal component associated and contrasting with a nonenhancing infundibular ependymal component; molecular grouping was not available then. Recently, Nagashima et $\mathrm{al}^{25}$ reported a case of a large suprasellar metastasis from a non-SHH/ non-WNT type, without further determination of group 3 or 4 . In our series, it was clearly significant that all tumors that presented with a metastasis in the anterior third ventricular infundibulum belonged to group 4 tumors. Besides medulloblastoma, suprasellar, third ventricular seeding is known to occur for high-grade gliomas and pineoblastomas. For some authors, the pattern of "bifocal germinoma" also corresponds to a pattern of metastatic disease spread. ${ }^{26}$ Therefore, it is not so much the suprasellar/anterior third ventricular location that is surprising in medulloblastoma but that this seemingly occurs in group 4 tumors and not in other groups. Another peculiarity of group 4 metastases is that they enhance in the subarachnoid space but not in the ventricles and that in the ventricles, they may even demonstrate no diffusion restriction. Metastases that do not enhance or show restricted diffusion would be easily missed in the leptomeninges, but not in the ventricles.

This study provides further indications that some of the imaging patterns of primary tumors and metastatic spread in medulloblastoma may be characteristic, though further studies may be needed. This finding is consistent with the idea that medulloblastomas of different groups are biologically different diseases, with different behavior and, possibly, distinct preferences for specific environments. The findings of group-specific patterns of metastatic disease spread complements the already described groupspecific radiologic features of the primary tumors.

The main limitation to this study is that it is retrospective; and because it covers a 17 -year period, it includes cases with very different MR imaging. Diffusion imaging was not routinely used during the earlier reviewed cases. Similarly, postcontrast FLAIR, which may allow detection of more leptomeningeal metastatic lesions, was not part of the MR imaging protocol during the period covered by this study. In addition, fewer spinal metastases detected in this study may be because we used only contrast-enhanced T1WI. The ongoing development of MR imaging sequences such as spine DWI and steady-state free precession could help in detecting more spinal metastases in the future.

\section{CONCLUSIONS}

In addition to imaging features of primary tumors, some of imaging patterns of metastatic dissemination in medulloblastoma seem characteristic, perhaps even specific to certain groups. This finding could further help in differentiating molecular groups, specifically groups 3 and 4 , when the characteristics of the primary tumor overlap.

\section{ACKNOWLEDGMENTS}

We thank our department of MR imaging Quality Specialist, Mr Manoj Singh, for the technical support in advanced MR imaging and protocols and our department Clinical Research Project Assistant, Ms Olga Carpio Pinto, for her support in editing this article.

\section{REFERENCES}

1. Taylor RE, Bailey CC, Robinson KJ, et al. Outcome for patients with metastatic (M2-3) medulloblastoma treated with SIOP/UKCCSG PNET-3 chemotherapy. Eur J Cancer 2005;41:727-34 CrossRef Medline

2. Zeltzer PM, Boyett JM, Finlay JL, et al. Metastasis stage, adjuvant treatment, and residual tumor are prognostic factors for medulloblastoma in children: conclusions from the Children's Cancer Group 921 randomized phase III study. J Clin Oncol 1999;17:832-45 CrossRef Medline

3. Gajjar A, Chintagumpala M, Ashley D, et al. Risk-adapted craniospinal radiotherapy followed by high-dose chemotherapy and stemcell rescue in children with newly diagnosed medulloblastoma (St 
Jude Medulloblastoma-96): long-term results from a prospective, multicentre trial. Lancet Oncol 2006;7:813-20 CrossRef Medline

4. Bouffet E. Embryonal tumours of the central nervous system. Eur J Cancer 2002;38:1112-20 CrossRef Medline

5. Torres CF, Rebsamen S, Silber JH, et al. Surveillance scanning of children with medulloblastoma. N Engl J Med 1994;330:892-95 CrossRef Medline

6. Cavalli FM, Remke M, Rampasek L, et al. Intertumoral heterogeneity within medulloblastoma subgroups. Cancer Cell 2017;31:73754.e6 CrossRef Medline

7. Ramaswamy V, Remke M, Adamski J, et al. Medulloblastoma subgroup-specific outcomes in irradiated children: who are the true high-risk patients? Neuro Oncol 2016;18:291-97 CrossRef Medline

8. Thompson EM, Hielscher T, Bouffet E, et al. Prognostic value of medulloblastoma extent of resection after accounting for molecular subgroup: a retrospective integrated clinical and molecular analysis. Lancet Oncol 2016;17:484-95 CrossRef Medline

9. Ramaswamy V, Taylor MD. Medulloblastoma: from myth to molecular. J Clin Oncol 2017;35:2355-63 CrossRef Medline

10. Patay Z, DeSain LA, Hwang SN, et al. MR imaging characteristics of wingless-type-subgroup pediatric medulloblastoma. AJNR Am J Neuroradiol 2015;36:2386-93. CrossRef Medline

11. Perreault S, Ramaswamy V, Achrol AS, et al. MRI surrogates for molecular subgroups of medulloblastoma. AJNR Am J Neuroradiol 2014;35:1263-69 CrossRef Medline

12. Northcott PA, Shih DJ, Remke M, et al. Rapid, reliable, and reproducible molecular sub-grouping of clinical medulloblastoma samples. Acta Neuropathol 2012;123:615-26 CrossRef Medline

13. Northcott PA, Korshunov A, Witt H, et al. Medulloblastoma comprises four distinct molecular variants. J Clin Oncol 2011;29: 1408-14 CrossRef Medline

14. Yeom KW, Mobley BC, Lober RM, et al. Distinctive MRI features of pediatric medulloblastoma subtypes. AJR Am J Roentgenol 2013; 200:895-903 CrossRef Medline

15. Gibson P, Tong Y, Robinson G, et al. Subtypes of medulloblastoma have distinct developmental origins. Nature 2010;468:1095-99 CrossRef Medline

16. Raybaud C, Ramaswamy V, Taylor MD, et al. Posterior fossa tumors in children: developmental anatomy and diagnostic imaging. Childs Nerv Syst 2015;31:1661-76 CrossRef Medline

17. Ramaswamy V, Remke M, Shih D, et al. Duration of the pre-diagnostic interval in medulloblastoma is subgroup dependent. Pediatr Blood Cancer 2014;61:1190-94 CrossRef Medline

18. Wu X, Northcott PA, Dubuc A, et al. Clonal selection drives genetic divergence of metastatic medulloblastoma. Nature 2012;482: 529-33 CrossRef Medline

19. Ramaswamy V, Remke M, Bouffet E, et al. Risk stratification of childhood medulloblastoma in the molecular era: the current consensus. Acta Neuropathol 2016;131:821-31 CrossRef Medline

20. Newton TH, Potts DG. Radiology of the Skull and Brain. Ventricles and Cisterns. Vol 4. St. Louis: C.V. Mosby; 1978

21. Fukusumi A, Maehara F, Hayashi T, et al. Two cases of cerebellar medulloblastoma associated with seeding in the suprasellar cistern [in Japanese]. Rinsho Hoshasen 1985;30:901-04 Medline

22. Blaser SI, Harwood-Nash DC. Neuroradiology of pediatric posterior fossa medulloblastoma. J Neurooncol 1996;29:23-34 CrossRef Medline

23. Shelton $\mathrm{CH}$ 3rd, Phillips CD, Laws ER, et al. Third ventricular lesion masquerading as suprasellar disease. Surg Neurol 1999;51:177-80 CrossRef Medline

24. Helton KJ, Gajjar A, Hill DA, et al. Medulloblastoma metastatic to the suprasellar region at diagnosis: a report of six cases with clinicopathologic correlation. Pediatr Neurosurg 2002;37:111-17 CrossRef Medline

25. Nagashima H, Nagashima T, Kawamura A, et al. Medulloblastoma with suprasellar solitary massive metastasis: case report. Neurol Neurochir Pol 2016;50:211-14 CrossRef Medline

26. Phi JH, Kim SK, Lee J, et al. The enigma of bifocal germ cell tumors in the suprasellar and pineal regions: synchronous lesions or metastasis? J Neurosurg Pediatr 2013;11:107-14 CrossRef Medline 